\title{
The instability of some non-full-support steady states in a random matching model of money
}

\author{
Pidong Huang*and Yoske Igarashi ${ }^{\ddagger}$
}

August 2, 2014

\begin{abstract}
Zhu (2003) shows existence of full-support monetary steady states with strictly concave value functions in a random matching model with individual money holdings in $\{0,1,2, \ldots, B\}$ for a general $B$. He also shows that corresponding to each such steady state is an $l$-replica steady state for each $l \in \mathbb{N}$ : money is traded in bundles of $l$ units, the support is $\{0, l, 2 l, \ldots, l B\}$, and the value function is a step-function with jumps at points of the support. We show that such $l$-replicas are unstable if the underlying full-support steady state is a pure strategy steady state and if the support of the initial distribution is not $\{0, l, 2 l, \ldots, l B\}$.

(JEL classification: C62, C78, E40)

Keywords: random matching model; monetary steady state; instability; Zhu (2003).
\end{abstract}

\section{Introduction}

Shi (1995) and Trejos-Wright (1995) study a matching model of fiat money with individual money holdings in the set $\{0,1\}$. Such holdings are special

\footnotetext{
*Korea University: pidonghuang@korea.ac.kr

${ }^{\dagger}$ University of Exeter: y.y.igarashi@exeter.ac.uk

${ }_{\ddagger}^{\ddagger}$ The authors are especially grateful to Neil Wallace for his guidance and encouragement. We also thank Daniella Puzzello, Ed Green, Ricardo Cavalcanti, Rulin Zhou, the editor and two anonymous referees, and the participants in the Cornell-Penn State macro workshop for their helpful comments and discussions. The title of the working paper version of this article was "Why Ten $\$ 1$ 's Are Not Treated as a $\$ 10 . "$
} 
because the distribution of holdings is determined by the stock of money; that is, it is unaffected by the trades that are made. That property disappears for any richer set of individual money holdings. For buyer take-it-or-leave-it offers, Zhu (2003) studies the model with individual money holdings in the set $\{0, \Delta, 2 \Delta, \ldots, B \Delta\}$, for arbitrary $B$. He provides sufficient conditions for the existence of a steady state with a full-support money-holding distribution and a strictly increasing and strictly concave value function, a steady state that we call a Zhu steady state.

In Zhu's model, there are three exogenous nominal quantities: $(\Delta, B \Delta, m)$, where $m$ is the per capita stock of money. If, for some positive integer $l \geq 2$, we compare that economy to an otherwise identical economy with nominal quantities $(l \Delta, l B \Delta, l m)$, then we have neutrality. But what if we compare $(\Delta, B \Delta, m)$ to $(\Delta, l B \Delta, l m)$ ? Zhu shows that any steady state for $(\Delta, B \Delta, m)$ is also a steady state for $(\Delta, l B \Delta, l m)$, one in which all owned/traded quantities of money are multiplied by $l$ and the value function is a step function with steps at and only at integer multiples of an $l$-bundle. We call such a non-full-support steady state an l-replica. In an $l$-replica with $l=10$, for example, ten $\$ 1$ 's are treated only as a bundle; that is, as a $\$ 10 .{ }^{1}$ Such a steady state implies a lower real balance of money and, almost certainly, lower welfare than in the full-support steady state of $(\Delta, l B \Delta, l m)$.

The presence of $l$-replicas complicates the use of the model for policy analysis unless there are reasons to ignore them. Wallace and Zhu (2004) show that such replicas are not robust to the introduction of a small utility of holding money. Here we show something even stronger; if $l$-replicas are constructed from a Zhu steady state that is supported by pure strategies, then they are not stable. Specifically, if the initial distribution has support different from $\{0, l \Delta, 2 l \Delta, \ldots, B l \Delta\}$, then there is no equilibrium that converges to such $l$-replicas.

Zhu steady states can either be pure-strategy steady states or mixed strategy steady states and, as just noted, our instability result applies only to $l$-replicas that are constructed from pure-strategy steady states. ${ }^{2}$ It remains an open question whether the result extends to $l$-replicas that are constructed from mixed-strategy steady states.

\footnotetext{
${ }^{1}$ The present paper is not about denomination of a currency. In particular, in Zhu's model a $\$ 10$ is nothing but $10 \$ 1$ 's because there is no carrying cost of money.

${ }^{2}$ See [4] for a demonstration that both kinds are generic. For any $B$, a pure-strategy Zhu steady state exists for a sufficiently high $\beta$. See Camera and Corbae (1999).
} 
One reason to study the Zhu (2003) model is that it has policy implications that differ from those of the model with money holdings in $\{0,1\}$ and from models with degenerate distributions of money holdings. In particular, as discussed in Wallace (2014) and shown by Molico (2006) and Deviatov (2006), moderate inflation improves welfare through re-distributional effects in versions of the Zhu model. That cannot happen in models with money holdings in $\{0,1\}$ or in models with degenerate distributions of money holdings.

\section{Model}

The model is that in Zhu (2003). Time is discrete, dated as $t \geq 0$. There is a non-atomic unit measure of infinitely-lived agents. There is a consumption good that is perfectly divisible and perishable. Each agent maximizes the expected discounted utility with discount factor $\beta \in(0,1)$. Utility in a period is $u(c)-q$, where $c \in \mathbb{R}_{+}$is the amount of good consumed and $q \in \mathbb{R}_{+}$is the amount of good produced. $u: \mathbb{R}_{+} \rightarrow \mathbb{R}$ is continuously differentiable, strictly increasing and strictly concave, and satisfies $u(0)=0$ and $u^{\prime}(\infty)=0$. In addition, $u^{\prime}(0)$ is sufficiently large but finite.

There is a fixed stock of intrinsically useless money that is indivisible and perfectly durable. Because of the neutrality we discussed in the previous section, the size of the smallest unit of money is normalized to one. Two other exogenous quantities are $(B, m)$, where $B$ is the maximum units that an agent can hold, and $m \in(0, B)$ is the fixed per capita stock of money. We denote the set of possible individual money holdings by $\mathbb{B}=\{0,1, \cdots, B\}$. The state of the economy at each date is a distribution over $\mathbb{B}$, which for each $k \in \mathbb{B}$ gives the fraction of agents who have $k$ units of money.

In each period, agents are randomly matched in pairs. With probability $1 / N$, where $N \geq 2$, an agent is a consumer (producer) and the partner is a producer (consumer). Such meetings are called single-coincidence meetings. With probability $1-2 / N$, the match is a no-coincidence meeting. ${ }^{3}$ In meetings, agents' money holdings are observable, but any other information about an agent's trading history is private.

In a single-coincidence meeting between a consumer with $i$ units of money

\footnotetext{
${ }^{3}$ One foundation is that there are $N$ types of agents and $N$ types of consumption goods, that type- $n$ agents can produce type- $n$ goods only and consume type- $(n+1)$ goods only, and that the money is symmetrically distributed across the types.
} 
and a producer with $j$ units of money, an $(i, j)$-meeting, the consumer makes a take-it-or-leave-it offer consisting of the amount to be produced, $q$, and the amount of money to be paid, $p$. The offer must be feasible, $0 \leq p \leq$ $\min \{i, B-j\}$, and must satisfy the producer's participation constraint, $-q+$ $\beta w_{j+p}^{t+1} \geq \beta w_{j}^{t+1}$, where $w_{k}^{t}$ is the expected discounted value of holding $k \in \mathbb{B}$ units of money, prior to date- $t$ matching. Because the optimal offer leaves no positive gain to the producer, the consumer's problem reduces to choosing $p$ in the feasible set of offers of money

$$
p^{t}\left(i, j, w^{t+1}\right)=\underset{0 \leq p \leq \min \{i, B-j\}}{\operatorname{argmax}}\left\{u\left(\beta w_{j+p}^{t+1}-\beta w_{j}^{t+1}\right)+\beta w_{i-p}^{t+1}\right\} .
$$

Because $p^{t}\left(i, j, w^{t+1}\right)$ is discrete and may be multi-valued, randomization over the elements of $p^{t}\left(i, j, w^{t+1}\right)$ is allowed. Let $\lambda^{t}(p ; i, j)$ be the probability that consumers with $i$ (pre-trade) in meetings with producers with $j$ offer $p$ at date $t$. It has support in $p^{t}\left(i, j, w^{t+1}\right)$ in equilibrium, so that

$$
\sum_{p \in p^{t}\left(i, j, w^{t+1}\right)} \lambda^{t}(p ; i, j)=1 .
$$

Let $\pi_{k}^{t}$ denote the fraction of agents holding $k$ units of money prior to date- $t$ matching. The law of motion is

$$
\begin{aligned}
\pi_{k}^{t+1}=\pi_{k}^{t} & +\frac{1}{N} \sum_{\{i, j \mid i>k\}} \pi_{i}^{t} \pi_{j}^{t} \lambda^{t}(i-k ; i, j) \\
& +\frac{1}{N} \sum_{\{i, j \mid j<k\}} \pi_{i}^{t} \pi_{j}^{t} \lambda^{t}(k-j ; i, j) \\
& -\frac{1}{N} \sum_{j} \pi_{k}^{t} \pi_{j}^{t} \sum_{p>0} \lambda^{t}(p ; k, j) \\
& -\frac{1}{N} \sum_{i} \pi_{i}^{t} \pi_{k}^{t} \sum_{p>0} \lambda^{t}(p ; i, k) .
\end{aligned}
$$

The Bellman equation is

$$
w_{i}^{t}=\frac{N-1}{N} \beta w_{i}^{t+1}+\frac{1}{N} \sum_{j=0}^{B} \pi_{j}^{t} \sum_{p} \lambda^{t}(p ; i, j)\left\{u\left(\beta w_{j+p}^{t+1}-\beta w_{j}^{t+1}\right)+\beta w_{i-p}^{t+1}\right\} .
$$


The first term on the r.h.s. corresponds to entering a no-coincidence meeting or becoming a producer who is indifferent between accepting and rejecting the offer. Free disposal of money is permitted which implies that the value function must be nondecreasing in every period:

$$
w_{i}^{t} \geq w_{i-1}^{t}, \text { for } i=1, \cdots B, \text { and } w_{0}^{t}=0 .
$$

Given (5), we focus on equilibria in which agents do not dispose of money.

Definition 1 Given $\pi^{0}$, an equilibrium is a sequence $\left\{\left(\lambda^{t}, \pi^{t}, w^{t}\right)\right\}_{t=0}^{\infty}$ that satisfies (1)-(5). A monetary steady state is $(\lambda, \pi, w)$ with $w \neq 0$ such that $\left(\lambda^{t}, \pi^{t}, w^{t}\right)=(\lambda, \pi, w)$ for all $t$ is an equilibrium. Pure-strategy steady states are those for which (1) has a unique solution for all meetings. Other steady states are called mixed-strategy steady states. ${ }^{4}$ A Zhu steady state is a steady state for which $\pi$ has a full support and $w$ is strictly increasing and strictly concave. ${ }^{5}$

Our definition of steady states differs from that in Zhu only in that we explicitly include $\lambda$, a description of trades. In a non-full support steady state, some meetings occur with zero probability. Our definition requires a definition of trades for all meetings, including those which occur with zero probability. That is because such meetings will in general occur with positive probability near a steady state.

Next, we formally define $l$-replicas for economies in which the smallest unit of money is one.

Definition 2 Let $s=(\hat{\lambda}, \hat{\pi}, \hat{w})$ be a Zhu steady state of economy $(B, m)$. For integer $l \geq 2$, an l-replica of $s$, denoted $s(l)=\left(\lambda^{*}, \pi^{*}, w^{*}\right)$, is a steady state of economy $(l B, l m)$ that satisfies

$$
\begin{aligned}
& \pi_{i l}^{*}=\hat{\pi}_{i}, \quad \text { and } \quad \pi_{i l+i^{\prime}}^{*}=0, \quad \forall i^{\prime} \in \mathbb{L}, \\
& w_{i l}^{*}=\hat{w}_{i}, \quad \text { and } \quad w_{i l+i^{\prime}}^{*}=w_{i l}^{*}, \quad \forall i^{\prime} \in \mathbb{L},
\end{aligned}
$$

where $\mathbb{L} \equiv\{1, \cdots, l-1\}$.

\footnotetext{
${ }^{4}$ Note that in this definition, the 'boundary' situations in which (1) has more than one solution but in which the randomization is degenerate are included in mixed-strategy equilibria. Such situations are non-generic.

${ }^{5}$ Zhu (2003) gives sufficient conditions for the existence of such steady states.
} 
When we discuss an $l$-replica or convergence to an $l$-replica, if an agent holds $i l+i^{\prime}$ units of money for some $i \in\{0,1, \cdots, B-1\}$ and $i^{\prime} \in \mathbb{L}$, we say that the agent has $i$ "bundles" and $i^{\prime}$ units of "change." 6

The following is our main result.

Proposition 1 Assume that $s(l)=\left(\lambda^{*}, \pi^{*}, w^{*}\right)$ is a steady-state that is an l-replica of a pure-strategy Zhu steady state. If $\pi^{0}$ has a support that is different from that of $\pi^{*}$, then there is no equilibrium such that $\left(\lambda^{t}, \pi^{t}, w^{t}\right) \rightarrow$ $\left(\lambda^{*}, \pi^{*}, w^{*}\right) .^{7}$

That is, if the initial distribution has some holdings of change, then the economy cannot converge to the $l$-replica steady state. Notice that our notion of stability requires convergence of trades. This seems reasonable because trades are what people do and what an outside observer would see. If the economy's distribution and value of money converge to those of $s(l)$ and agents' actions converge, then we must have $\lim _{w \rightarrow \bar{w}} p(i, j ; w) \subset p(i, j ; \bar{w})$. For this reason, the description of optimal trades in $l$-replicas, including those for meetings that occur with zero probability, is relevant.

The standard approach to stability analysis of difference equation systems (see, for example, [7]) is to compare the number of eigenvalues of the dynamical system that are strictly smaller than one in absolute value, say $a$, and the number of initial conditions, say $b$. If $a=b(a>b)$, then there is a unique (an infinity of) convergent path(s). If $a<b$, then there is no convergent solution. Our analysis must go beyond those results for two reasons. First, our dynamical system necessarily involves unit root convergence. Second, the $l$-replica steady state is on the boundary of the state space in two senses: $\pi^{*}$ does not have full support and $w^{*}$ is not strictly increasing. Both play important parts in our proof.

\section{A simple example}

We start with an example in which $B=1$ (i.e., Trejos-Wright) and $l=2$. This example is simple because it is a pure-strategy steady state and because

\footnotetext{
${ }^{6}$ In what follows, non-prime letters $(i, j$, etc.) indicate numbers of bundles, and letters with primes $\left(i^{\prime}, j^{\prime} \in \mathbb{L}\right.$, etc.) indicate units of change.

${ }^{7}$ The fact that the Zhu steady state has a strictly concave value function is used only in Lemma 1 and it can be dropped at the cost of complicating the proof. Because it is not known whether a full-support steady state with a non-concave value function exists, we do not present that extension here.
} 
the trades in the $l=2$ replica are easy to describe. In addition, the aggregate state of the $l=2$ replica is one-dimensional and can be described by the fraction of people with one unit of money. Despite its simplicity, it captures some of the main ingredients of our stability analysis. The proof is by way of contradiction and shows that any convergent path violates either the consumer's optimality conditions (1) or monotonicity of the value function (5).

Because $s(2) \equiv\left(\lambda^{*}, \pi^{*}, w^{*}\right)$ is identical to the Trejos-Wright steady state, we have $\lambda^{*}(2 ; 2,0)=1,\left(\pi_{0}^{*}, \pi_{2}^{*}\right)=(1-m, m), w_{0}^{*}=0$, and $w_{2}^{*}$ is the unique positive solution to

$$
\left(\frac{N / \beta-N}{1-m}+1\right) \beta w_{2}^{*}=u\left(\beta w_{2}^{*}\right)
$$

where the positive solution exists if and only if the Trejos-Wright steady state for $B=1$ economy exists.

Also, (6) and (7) imply $\pi_{1}^{*}=0$ and $w_{1}^{*}=0$. Given such $w_{1}^{*}$ and $w_{2}^{*}$, it follows that $\lambda^{*}(1 ; 1,1)=1$ and $\lambda^{*}(1 ; 2,1)=1 .^{8}$

Assume $\pi_{1}^{0}>0$ and, by way of contradiction, that there exists an equilibrium $\left(\lambda^{t}, \pi^{t}, w^{t}\right)$ that converges to an $l$-replica for $l=2$, denoted $\left(\lambda^{*}, \pi^{*}, w^{*}\right)$. We next show that $\lambda^{*}(1 ; 1,0)=1$. If the economy is close to $\left(\pi^{*}, w^{*}\right)$, then (4) and $\pi_{1}^{0}>0$ imply $w_{1}^{0}>0$ because there is a positive probability that a consumer with one unit meets a producer with one unit and the consumer can get a positive amount of utility from such a meeting. Because equation (8) implies $u(x)>x$ for all $x<\beta w_{2}^{*}$, we have $u\left(\beta w_{1}^{t}\right)>\beta w_{1}^{t}$. Therefore, $\lambda^{t}(1 ; 1,0)=1$ is the only possibility for such a convergent equilibrium path. We call such payment strategy described in Table 1 Payment Strategy 1.

Using $\pi_{0}+\pi_{1}+\pi_{2}=1$ and $0 \pi_{0}+1 \pi_{1}+2 \pi_{2}=2 \mathrm{~m}$, the money-holding distribution can be characterized by $\pi_{1}^{t}$ only:

$$
\left(\pi_{0}^{t}, \pi_{1}^{t}, \pi_{2}^{t}\right)=\left(1-m-\frac{\pi_{1}^{t}}{2}, \quad \pi_{1}^{t}, \quad m-\frac{\pi_{1}^{t}}{2}\right) .
$$

Let $\pi_{1}^{t} \in[0,2 \min (m, 1-m)]$ to ensure $\pi_{0}^{t}, \pi_{2}^{t} \in[0,1]$. Under Payment Strategy 1 , the law of motion is

$$
\pi_{1}^{t+1}=\Psi\left(\pi_{1}^{t}\right) \equiv \pi_{1}^{t}-\frac{2\left(\pi_{1}^{t}\right)^{2}}{N}
$$

\footnotetext{
${ }^{8}$ One can show that the step function form (7) of the value function in our definition of $l$-replicas is in fact necessary in this example.
} 
Table 1: Payment Strategy 1

\begin{tabular}{|c|c|c|c|c|}
\hline \multicolumn{2}{|c|}{} & \multicolumn{3}{|c|}{ Seller's money holding } \\
\cline { 3 - 5 } \multicolumn{2}{c|}{} & 0 & 1 & 2 \\
\hline \multirow{2}{*}{$\begin{array}{c}\text { Buyer's } \\
\text { money } \\
\text { holdings }\end{array}$} & 0 & - & - & - \\
\cline { 2 - 5 } & 1 & 1 unit & 1 unit & - \\
\cline { 2 - 5 } & 2 & 2 units & 1 unit & - \\
\hline
\end{tabular}

Figure ?? shows the law of motion. The law of motion is locally stable at $\pi_{1}^{*}=0$, but it exhibits unit root convergence: the slope at the fixed point is one. This happens because there are no net inflows into holdings of one unit and there are net outflows, but only from $(1,1)$-meetings. The unit root arises because as $\pi_{1}^{t}$ goes to zero, the frequency of $(1,1)$-meetings, which is equal to $\left(\pi_{1}^{t}\right)^{2} / N$, goes to zero much faster.

Under Payment Strategy 1, the Bellman equation is

$$
\left\{\begin{array}{l}
w_{1}^{t}=\frac{N-1+\pi_{2}^{t}}{N} \beta w_{1}^{t+1}+\frac{\pi_{0}^{t}}{N} u\left(\beta w_{1}^{t+1}\right)+\frac{\pi_{1}^{t}}{N} u\left(\beta w_{2}^{t+1}-\beta w_{1}^{t+1}\right) \\
w_{2}^{t}=\frac{N-1+\pi_{2}^{t}}{N} \beta w_{2}^{t+1}+\frac{\pi_{0}^{t}}{N} u\left(\beta w_{2}^{t+1}\right)+\frac{\pi_{1}^{t}}{N}\left[u\left(\beta w_{2}^{t+1}-\beta w_{1}^{t+1}\right)+\beta w_{1}^{t+1}\right]
\end{array} .\right.
$$

The Jacobian of the r.h.s. of (11) with respect to $w^{t+1}=\left(w_{1}^{t+1}, w_{2}^{t+1}\right)$ evaluated at the $l=2$ replica is

$$
\left[\begin{array}{ll}
\bar{\lambda} & 0 \\
0 & \underline{\lambda}
\end{array}\right]
$$

where

$$
\begin{aligned}
& \bar{\lambda} \equiv \frac{\pi_{0}^{*}}{N} u^{\prime}(0) \beta+\frac{N-1+\pi_{2}^{*}}{N} \beta>1 \\
& \underline{\lambda} \equiv \frac{\pi_{0}^{*}}{N} u^{\prime}\left(\beta w_{2}^{*}\right) \beta+\frac{N-1+\pi_{2}^{*}}{N} \beta \in(0,1) .
\end{aligned}
$$

If (8) has a positive solution, then (13) and (14) hold. Because the matrix (12) has an inverse and because we can extend the domain of $u$ to include an open neighborhood around 0, the implicit function theorem can be applied to (11) to get

$$
w^{t+1}=\Phi\left(\pi^{t}, w^{t}\right)
$$


which we refer to as the forward-looking Bellman equation and which is valid in the neighborhood of an $l=2$ replica.

Equations (10) and (15) form our dynamical system. The Jacobian matrix of the joint system evaluated at the $l=2$ replica is

$$
\left[\begin{array}{cc}
\Psi_{\pi} & O \\
\Phi_{\pi} & \Phi_{w}
\end{array}\right]=\left[\begin{array}{ccc}
1 & 0 & 0 \\
-\gamma / \bar{\lambda} & 1 / \bar{\lambda} & 0 \\
-\delta /(2 \underline{\lambda}) & 0 & 1 / \underline{\lambda}
\end{array}\right]
$$

where

$$
\begin{aligned}
\gamma & \equiv \frac{1}{N} u\left(\beta w_{2}^{*}\right)>0 \\
\delta & \equiv \frac{1}{N}\left[u\left(\beta w_{2}^{*}\right)-\beta w_{2}^{*}\right]>0,
\end{aligned}
$$

and where the last inequality follows from (8).

Since, initially, $\pi_{1}^{0} \neq 0$ and the law of motion has unit root convergence, the convergence trajectory will eventually be parallel to the eigenspace of (16) associated with the unit eigenvalue ${ }^{9}$. The associated eigenvector, which constitutes a base of the space, has the form

$$
\left[\begin{array}{c}
1 \\
\frac{-\gamma}{\lambda-1} \\
*
\end{array}\right]
$$

where $*$ is a number irrelevant to our argument. Note that $-\gamma /(\bar{\lambda}-1)<0$. Since the first variable of the linearized system, $\pi_{1}^{t}-\pi_{1}^{*}$, is always positive in the process of convergence, the second variable, $w_{1}^{t}-w_{1}^{*}$ (and therefore $w_{1}^{t}$ ) will eventually become negative, which violates (5). We conclude that there is no convergent equilibrium path.

We have established a non-existence result for some class of dynamic equilibria. Here we will try to give some intuitive explanation for this nonexistence. Why is it impossible that people follow Payment Strategy 1, believing that $\left(w_{1}^{t}, w_{2}^{t}\right)$ will keep positive value but eventually converge to $\left(0, w_{2}^{*}\right)$ ? In that case, convergence of $w_{1}^{t}$ to zero should be exponential and cannot be a unit-root convergence; for a fiat money to ever have positive value, $u^{\prime}(0)$ cannot be too small, which implies high marginal utility of very

\footnotetext{
${ }^{9}$ See Subsection "dominant eigenvector" on page 165 of [6].
} 
little consumption and necessitates quick convergence of $w_{1}^{t}$ to zero ${ }^{10}$. On the other hand, $\pi_{1}^{t}$ goes to zero extremely slowly, resembling a positive constant in the end. These two facts are not consistent with each other because if there is non-negligible chance of meeting a producer with one unit of money, the value of holding one unit of money is not negligible either.

Given that such equilibrium paths do not exist, people should not believe them. They should believe something else, for example, converging to a nonmonetary steady state or to a full-support steady state. In the latter case, following Payment Strategy 1 would no longer be optimal. ${ }^{11}$

\section{Proof for the general case}

The argument for the $B=1, l=2$ example uses knowledge of the trades in the pure-strategy Zhu steady state. In the general case, we know very little about those trades. Nevertheless, as we now show, a proof can be constructed using similar ideas. The proof is by way of contradiction so we suppose that there exists a sequence $\left\{\lambda^{t}, \pi^{t}, w^{t}\right\}$ that converges to an $l$-replica steady state and examine the implications of the resulting dynamical system. The proof relies on five lemmas. ${ }^{12}$

The first lemma describes features of optimal trades in $s(l)$, including features of trades in some of the zero-probability meetings.

Lemma 1 Suppose that $s=(\hat{\lambda}, \hat{\pi}, \hat{w})$ is a pure-strategy Zhu steady state and let $\hat{p}(i, j)$ be the unique solution of (1). Then the trade of $s(l)$ satisfies the following: in $\left(i l+i^{\prime}, j l+j^{\prime}\right)$-meetings,

(i) if $i^{\prime}+j^{\prime}<l$, then after the meeting, consumer has $i-\hat{p}(i, j)$ bundles and producer has $j+\hat{p}(i, j)$ bundles; and

(ii) if $i^{\prime}+j^{\prime} \geq l$, then after the meeting, consumer has $i+1-\max (1, \hat{p}(i+1, j))$ bundles and producer has $j+\max (1, \hat{p}(i+1, j))$ bundles.

Although change has zero value at $s(l)$, when the sum of change in a meeting exceeds $l$ (case (ii)), the producer can form an extra bundle by receiving enough change from the consumer. Therefore, when the total amount

\footnotetext{
${ }^{10}$ This reason is similar to why the convergence to a non-monetary steady state is quick for some models of fiat money (e.g., Diamond's OLG model of money, or the Trejos-Wright model).

${ }^{11}$ For the two-unit bound case, [4] shows that full-support steady states always exist and is almost always locally stable.

${ }^{12}$ The proofs of the lemmas are in the Appendix.
} 
of change in a single-coincidence meeting exceeds $l$, the consumer behaves as if he had an extra bundle.

Before proceeding to the other lemmas, it is helpful to rearrange components in the $\lambda^{t}, \pi^{t}$ and $w^{t}$ vectors. First, we let $\lambda^{t}$ include only trades of meetings in which either consumer or producer has positive amount of change. If neither has change, then the consumer pays $\hat{p}(i, j) l$ with probability one, so there is no randomization. Note also that one element of each $\lambda^{t}\left(\cdot ; i l+i^{\prime}, j l+j^{\prime}\right)$ is eliminated using $(2)$.

Next, we eliminate $\pi_{0}^{t}$ and $\pi_{B l}^{t}$ in $\pi^{t}$ using the following adding-up conditions:

$$
\sum_{i=0}^{B l} \pi_{i}^{t}=1, \quad \text { and } \quad \sum_{i=0}^{B l} i \pi_{i}^{t}=l m .
$$

Then the remaining components of $\pi^{t}$ are divided into $l$ groups, where each group consists of $\pi_{i}$ 's that have the same amount of change. That is, we now let the state be described by $\left(\pi_{N}^{t}, \pi_{F}^{t}\right)$, where $\pi_{N}^{t} \equiv\left(\pi^{t}\left(i^{\prime}\right)\right)_{i^{\prime} \in \mathbb{L}}$ with $\pi^{t}\left(i^{\prime}\right) \equiv\left(\pi_{i l+i^{\prime}}^{t}\right)_{i \in \mathbb{B} \backslash B}$, and $\pi_{F}^{t} \equiv\left(\pi_{i l}^{t}\right)_{i \in \mathbb{B} \backslash\{0, B\}}$. As regards $w^{t}$, we use the incremental value of change, $w_{i l+i^{\prime}}^{t}-w_{i l}^{t}$ and group them into $l-1$ groups according to the amount of change. Let $\Delta w^{t} \equiv\left(\Delta w_{i^{\prime}}^{t}\right)_{i^{\prime} \in \mathbb{L}}$, where $\Delta w_{i^{\prime}}^{t} \equiv$ $\left(w_{i l+i^{\prime}}^{t}-w_{i l}^{t}\right)_{i \in \mathbb{B} \backslash\{B\}}$ and let $w_{F}^{t}=\left(w_{i l}^{t}\right)_{i \in \mathbb{B}}$. Notice that $w^{t}$ can be recovered from $\left(\Delta w^{t}, w_{F}^{t}\right)$. The one-to-one transformation of variables from $\left(\pi^{t}, w^{t}\right)$ to $\left(\pi_{N}^{t}, \Delta w^{t}, \pi_{F}^{t}, w_{F}^{t}\right)$ turns out to be useful because, as will be shown in Lemmas 2 and 3, the Jacobians of law of motion and Bellman equation come to have tractable triangular forms. In addition, the linearized system for $\left(\pi_{N}^{t}, \Delta w^{t}\right)$ does not depend upon the other two variables. In other words, these two variables are locally autonomous.

We express the law of motion and the Bellman equation in terms of the transformed variables as

$$
\left(\pi_{N}^{t+1}, \pi_{F}^{t+1}\right)=\Psi\left(\pi_{N}^{t}, \pi_{F}^{t}, \lambda^{t}\right)
$$

and

$$
\left(\Delta w^{t}, w_{F}^{t}\right)=\phi\left(\pi_{N}^{t}, \Delta w^{t+1}, \pi_{F}^{t}, w_{F}^{t+1}, \lambda^{t}\right)
$$

We let $\Psi$ and $\phi$ with superscripts refer to each component of the mappings and those with subscripts refer to derivatives with respect to that subscript variable. For example $\phi^{\Delta w}$ indicates the subvector that defines $\Delta w^{t}$.

The next lemma describes properties of the Jacobian of $\Psi$, the most important of which concerns the existence of unit eigenvalues. 
Lemma 2 (i) The Jacobian of $\Psi$ with respect to $\pi$ evaluated at $\left(\lambda^{*}, \pi^{*}\right)$ has the following triangular form:

$$
\Psi_{\pi}=\left[\begin{array}{cc}
\Psi_{\pi_{N}}^{\pi_{N}} & O \\
\Psi_{\pi_{N}}^{\pi_{F}} & \Psi_{\pi_{F}}^{\pi_{F}}
\end{array}\right] \text { with } \Psi_{\pi_{N}}^{\pi_{N}}=\left[\begin{array}{ccc}
\Psi_{\pi(1)}^{\pi(1)} & \cdots & \Psi_{\pi(l-1)}^{\pi(1)} \\
O & \ddots & \vdots \\
O & O & \Psi_{\pi(l-1)}^{\pi(l-1)}
\end{array}\right]
$$

where $\Psi_{\pi\left(v^{\prime}\right)}^{\pi\left(k^{\prime}\right)}=\left(\frac{\partial \pi_{k l+k^{\prime}}^{t+1}}{\partial \pi_{v l+v^{\prime}}^{t}}\right)_{k, v=0,1, \cdots, B-1}$.

(ii) $\Psi_{\pi_{N}}^{\pi_{N}}$ has at least one unit eigenvalue.

(iii) If $\Psi_{\pi_{N}}^{\pi_{N}}$ has a positive eigenvalue that is strictly smaller than one, then the associated eigenvector has both strictly positive and strictly negative elements.

(iv) $\Psi_{\lambda}=0$.

The proof of the above lemma involves studying the derivative of $\Psi$. Part (i) derives the Jacobian by straightfoward differentiation. Parts (ii) and (iii) show that the convergence of $\pi_{N}^{t}$ to zero necessarily involves a unit-root. The intuition is analogous to that of the $\{0,1,2\}$ case; In order for the measure of people holding positive change to go to zero, it is necessary for them to be matched with others with change. However, as the measure of people with change goes to zero, the frequency of such meetings goes to zero much faster. Part (iv) is also a straightforward differentiation and shows that the small change in $\lambda^{t}$ does not affect $\pi_{N}^{t}$ near the l-replica.

We now investigate the Jacobians of $\phi$, equation (21).

Lemma 3 (i) The linear approximation of $\phi^{\Delta w}$ around the l-replica has the form

$$
\Delta w^{t}=\phi_{\Delta w}^{\Delta w} \Delta w^{t+1}+\phi_{\pi_{N}}^{\Delta w} \pi_{N}^{t} .
$$

That is, it does not depend on $\pi_{F}^{t}, w_{F}^{t+1}$, or $\lambda^{t}$.

(ii) The Jacobian $\phi_{\pi_{N}}^{\Delta w}$ consists of $(l-1) \times(l-1)$ blocks and has a lower-right triangular form:

$$
\phi_{\pi_{N}}^{\Delta w}=\left[\begin{array}{cccc}
O & \cdots & O & K \\
\vdots & / & / & \vdots \\
O & / & & \vdots \\
K & \cdots & \cdots & K
\end{array}\right]
$$


where $K$ is a $B$ by $B$ matrix that only has strictly positive elements. (iii) The Jacobian $\phi_{\Delta w}^{\Delta w}$ has a lower-left triangular form:

$$
\phi_{\Delta w}^{\Delta w}=\left[\begin{array}{cccc}
\phi_{\Delta w_{1}}^{\Delta w_{1}} & O & \cdots & O \\
\vdots & \ddots & \ddots & \vdots \\
\vdots & & \ddots & O \\
\phi_{\Delta w_{1}}^{\Delta w_{l-1}} & \cdots & \cdots & \phi_{\Delta w_{l-1}}^{\Delta w_{l-1}}
\end{array}\right]
$$

where each diagonal block $\phi_{\Delta w_{i^{\prime}}}^{\Delta w_{i^{\prime}}}$ is a B by B matrix that only has nonnegative elements.

For the purpose of deriving the Jacobian of (21), we do not need the explicit form of (21); because the change of variables from $w^{t}$ to $\left(\Delta w^{t}, w_{F}^{t}\right)$ is a linear transformation, we can first linearize the original Bellman equation and then do the variable transformation. That way, we get the linear expansion of (21) or, equivalently, the Jacobian of (21), which has several properties as the lemma summarizes.

We are now ready to obtain a stacked system and study its Jacobian. We apply the implicit function theorem to solve for $\left(\Delta w^{t+1}, w_{F}^{t+1}\right)$ as a function of $\left(\lambda^{t}, \pi^{t}, w^{t}\right)$ from Bellman equation (21) around the $l$-replica and refer to it as the forward-looking Bellman equation, denoted by $\Phi$. Then we stack the law of motion and the forward-looking Bellman equation:

$$
\begin{aligned}
\left(\pi_{N}^{t+1}, \pi_{F}^{t+1}\right) & =\Psi\left(\pi_{N}^{t}, \pi_{F}^{t}, \lambda^{t}\right) \\
\left(\Delta w^{t+1}, w_{F}^{t+1}\right) & =\Phi\left(\pi_{N}^{t}, \Delta w^{t}, \pi_{F}^{t}, w_{F}^{t}, \lambda^{t}\right) .
\end{aligned}
$$

The next lemma shows that $\left(\pi_{N}^{t}, \Delta w^{t}\right)$ does not depend on the other variables in the linearized system (that is, $\left(\pi_{N}^{t}, \Delta w^{t}\right)$ is locally autonomous) and gives the Jacobian of the linear system of $\left(\pi_{N}^{t}, \Delta w^{t}\right)$.

Lemma 4 In the linearized system of (26)-(27), $\left(\pi_{N}^{t+1}, \Delta w^{t+1}\right)$ depends only upon $\left(\pi_{N}^{t}, \Delta w^{t}\right)$ and does not depend on $\left(\pi_{F}^{t}, w_{F}^{t}, \lambda^{t}\right)$. The Jacobian of the linearized system for $\left(\pi_{N}^{t}, \Delta w^{t}\right)$ is given by

$$
A \triangleq\left[\begin{array}{cc}
\Psi_{\pi_{N}}^{\pi_{N}} & O \\
-\left[\phi_{\Delta w}^{\Delta w}\right]^{-1} \phi_{\pi_{N}}^{\Delta w} & {\left[\phi_{\Delta w}^{\Delta w}\right]^{-1}}
\end{array}\right]
$$

Moreover, A has a unit eigenvalue. 
The proof uses Lemmas 2 and 3.

Matrix $A$ represents the linearized system of $\left(\pi_{N}^{t}, \Delta w^{t}\right)$. It turns out that a unit eigenvalue(s) of $A$ dictates the dynamics of $\left(\pi_{N}^{t}, \Delta w^{t}\right)$. The following lemma characterizes associated eigenvectors. Such an eigenvector is relevant to our analysis because it represents the trajectory of convergence of $\left(\pi_{N}^{t}, \Delta w^{t}\right)$ to zero.

Lemma 5 Denote an eigenvector of $\Psi_{\pi_{N}}^{\pi_{N}}$ associated with a unit eigenvalue by $z=\left(z_{1}, \cdots, z_{l-1}\right) \geq 0$, where each $z_{i^{\prime}}$ is a B-dimensional column vector. Let $\varphi_{\Delta w_{i^{\prime}}} \triangleq\left(I-\phi_{\Delta w_{i^{\prime}}}^{\Delta w_{i^{\prime}}}\right)^{-1} K z_{l-i^{\prime}}$, with $i^{\prime} \in \mathbb{L}$.

(i) The eigenvector of matrix $A$ associated with a unit eigenvalue has the form $\left(z, \varpi_{1}, \cdots, \varpi_{l-1}\right)$, with

$$
\left[\begin{array}{c}
\varpi_{1} \\
\vdots \\
\varpi_{l-1}
\end{array}\right]=\left[\begin{array}{c}
0 \\
\vdots \\
0 \\
\varphi_{\Delta w_{l-1}}
\end{array}\right]+\left[\begin{array}{c}
0 \\
\vdots \\
\varphi_{\Delta w_{l-2}} \\
*
\end{array}\right]+\cdots+\left[\begin{array}{c}
\varphi_{\Delta w_{1}} \\
* \\
\vdots \\
*
\end{array}\right] .
$$

(ii) If $z_{l-i^{\prime}} \geq 0, z_{l-i^{\prime}} \neq 0$ and $\varphi_{\Delta w_{i^{\prime}}} \geq 0$, then $\varphi_{\Delta w_{i^{\prime}}}>0$.

(iii) If $z_{l-i^{\prime}} \geq 0, z_{l-i^{\prime}} \neq 0$ and $\lambda^{*}\left(i^{\prime} ; i^{\prime}, 0\right)=1$, then $\varphi_{\Delta w_{i^{\prime}}}$ has strictly negative elements.

The proof is matrix algebra in which the lower triangular form of (29) is from the triangular forms of (24) and (25). Part (ii) is from the fact that $K$ is a strictly positive matrix and implies that holding $i^{\prime}$ units of change can have positive value if positive measure of people have $l-i^{\prime}$ units of change. Part (iii) provides a sufficient condition under which the value of $i^{\prime}$ units of change has strictly negative elements. This will lead to a contradiction in the proof of Proposition 1.

Proof of Proposition 1. Suppose by way of contradiction that there exists an equilibrium path $\left(\lambda^{t}, \pi^{t}, w^{t}\right)$ convergent to an $l$-replica of a pure-strategy Zhu steady state, $\left(\lambda^{*}, \pi^{*}, w^{*}\right)$. By assumption, the initial distribution has a different support from $\pi^{*}$ so that $\pi_{N}^{0} \neq 0$. Then, (4) implies $\Delta w^{0} \neq 0$ because some agents with positive amount of change at the initial date has chance to meet a producer with change and get extra amount of goods by transfering their change. Note also that because $\left(\pi_{N}^{*}, \Delta w^{*}\right)=(0,0)$, it should be the case that $\left(\pi_{N}^{t}-\pi_{N}^{*}, \Delta w^{t}-\Delta w^{*}\right) \geq 0$ for all $t \geq 0$. 
The sequence must satisfy (3), (4) and, if randomization occurs, the indifference across solutions (1). By Lemma 4, sufficiently near the steady state, $\left(\pi_{N}^{t}, \Delta w^{t}\right)$ forms a locally autonomous system (i.e., does not depend on other variables) and the linear system is represented by the Jacobian (28). In what follows we show that the dominant eigenvalue that characterizes the convergence of $\left(\pi_{N}^{t}, \Delta w^{t}\right)$ is a unit eigenvalue and not an eigenvalue that is smaller than one.

Suppose by way of contradiction that the dominant eigenvalue is strictly smaller than one. It is enough to consider the evolution of $\pi_{N}^{t}$ in this case. By Lemma 2 (iii), the associated eigenvector of $\Psi_{\pi_{N}}^{\pi_{N}}$ has strictly negative elements. The dominant mode argument (see [6]) implies that $\pi_{N}^{t}$ eventually becomes parallel to the associated eigenvector. It follows that some elements in $\pi_{N}^{t}$ will become strictly negative, contradicting $\pi_{N}^{t} \geq 0$.

Therefore the law of motion involves unit-root convergence, so does the dynamics of $\left(\pi_{N}^{t}, \Delta w^{t}\right)$. The dominant mode argument implies that $\left(\pi_{N}^{t}, \Delta w^{t}\right)$ eventually becomes parallel to an eigenspace, vectors of which have the form of $\left(z, \varpi_{1}, \cdots, \varpi_{l-1}\right)$ in Lemma 5 . It follows that $\left(z, \varpi_{1}, \cdots, \varpi_{l-1}\right)$ is nonnegative because $\left(\pi_{N}^{t}-\pi_{N}^{*}, \Delta w^{t}-\Delta w^{*}\right)$ is nonnegative along the convergent path. Let $h^{\prime}=\max \left\{i^{\prime} \mid z_{i^{\prime}} \neq 0\right\}$. By Lemma 5(i), we have

$$
\left[\begin{array}{c}
\varpi_{1} \\
\vdots \\
\varpi_{l-1}
\end{array}\right]=\left[\begin{array}{c}
0 \\
\vdots \\
0 \\
\varphi_{\Delta w_{l-1}}
\end{array}\right]+\cdots+\left[\begin{array}{c}
0 \\
\vdots \\
\varphi_{\Delta w_{l-h^{\prime}}} \\
*
\end{array}\right] .
$$

In the following, let $w_{x+p}-w_{x} \equiv \Delta w(x, p)$.

Case 1: Suppose $h^{\prime}<l-1$. Equation (30) implies $0 \leq \varpi_{l-h^{\prime}}=\varphi_{\Delta w_{l-h^{\prime}}}$ and $\varpi_{l-i^{\prime}}=0$ for $i^{\prime}>h^{\prime}$. By Lemma 5(ii), $\varphi_{\Delta w_{l-h^{\prime}}} \geq 0$ implies $\varphi_{\Delta w_{l-h^{\prime}}}>0$ and hence $\varpi_{l-h^{\prime}}>0$. With the dominant mode argument, $\Delta w^{t}$ becomes parallel to $\left(0, \cdots, 0, \varpi_{l-h^{\prime}}, \cdots, \varpi_{l-1}\right)$. In other words, the value of less than $l-h^{\prime}$ units of change will become negligible compared with the values of $l-h^{\prime}$ units of change. Furthermore, the fact that $\Delta w^{t}\left(0, l-h^{\prime}\right)$ is strictly positive and converges to zero implies $u^{\prime}(0) \beta \Delta w^{t}\left(0, l-h^{\prime}\right)>\beta \Delta w^{t}\left(0, l-h^{\prime}\right)$. (That is, $u\left(\beta w_{l-h^{\prime}}^{t}-\beta w_{0}^{t}\right)>\beta w_{l-h^{\prime}}^{t}$ once $w_{l-h^{\prime}}^{t}$ gets sufficiently close to zero.) Hence, the value of trading all the change dominates the value of no-trade in $\left(l-h^{\prime}, 0\right)$-meetings. Therefore, we have $\lambda^{*}\left(l-h^{\prime} ; l-h^{\prime}, 0\right)=1$. Lemma 5(iii) implies that $\varphi_{\Delta w_{l-h^{\prime}}}$ has negative elements, a contradiction to $\varphi_{\Delta w_{l-h^{\prime}}} \geq 0$. Case 2: Suppose $h^{\prime}=l-1$. Arguments similar to those of case 1 lead to 
$\lambda^{*}(1 ; 1,0)=1$. Lemma 5 (iii) implies that $\varphi_{\Delta w_{1}}$ has negative elements, which contradicts to $\varphi_{\Delta w_{1}} \geq 0$.

\section{Concluding remarks}

This paper shows instability of the non-full-support steady states that are l-replicas of pure-strategy Zhu (2003) full-support steady states. A possible extension is to consider the case where the Zhu full-support steady state is a mixed-strategy steady state. In order to do that, bundle-trading strategies in $\left(v l+v^{\prime}, j l\right)$-meetings would have to be allowed to vary with $v^{\prime} \in L$. A consequence is that the linearized Bellman equation for $\Delta w^{t}$ in Lemma 3 would depend on $w_{F}$. In such a case, a different proof is needed.

A natural question is whether our instability result holds under alternative assumptions. For example, does it hold if money is divisible? Does it hold in a version with lotteries?

Another relevant issue is stability of steady states more generally. Lomeli and Temzelides [5] show in the $B=1$ case that the non-monetary steady state is stable and indeterminate. In a companion paper [4], we provide a stability analysis of full-support monetary steady states for the $B=2$ case. We show that the full-support steady states in the $\{0,1,2\}$ economy are locally stable, whether they are pure-strategy or mixed-strategy steady states. Apart from that, there is no stability analysis for full-support steady states for $B>1$.

There is very little work on stability of steady states in matching models of money. An exception is Green and Zhou (2002), who also study a dynamic equilibrium in a matching model of fiat money. However, the value of money in their stationary equilibrium is linear so that 'change' in their stationary equibrium is not worthless as it is in an l-replica of a Zhu steady state. Also, the models are very different.

\section{Appendix}

Proof of Lemma 1. The optimality of the bundle trading specified in (i) is obvious and proof is omitted. For the optimality of (ii), note first that under $i^{\prime}+j^{\prime} \geq l$, the consumer pays at least $l-j^{\prime}\left(\leq i^{\prime}\right)$ units in order for the producer to form one bundle and produce a positive amount of goods. 
Suppose that the consumer pays another $s$ bundles and $s^{\prime}$ units of change to the producer such that $\left(s, s^{\prime}\right)$ solves the following.

$$
\begin{aligned}
\max _{\substack{s \in\{0,1, \cdots, \min (i, B-j-1)\} \\
s^{\prime} \in\left\{0, \cdots, i^{\prime}+j^{\prime}-l\right\} \\
(j+1) l+s l+s^{\prime} \leq B l}} u\left[\beta\left(w_{(j+1) l+s l+s^{\prime}}^{*}-w_{j l+j^{\prime}}^{*}\right)\right]+\beta w_{i l+i^{\prime}-\left(l-j^{\prime}\right)-\left(s l+s^{\prime}\right)}^{*} \\
=\max _{s+1 \in\{1, \cdots, \min (i+1, B-j)\}} u\left[\beta\left(w_{j l+(s+1) l}^{*}-w_{j l}^{*}\right)\right]+\beta w_{((i+1)-(s+1)) l}^{*}
\end{aligned}
$$

where the equality follows from the step-function form (7). If $\hat{p}(i+1, j)$ is not equal to zero, then $s+1$ is equal to $\hat{p}(i+1, j)$.

If $\hat{p}(i+1, j)=0$, then $s+1=0$ is not possible. In this case, we will consider any second best solution. Given that the objective function in (31) is concave in $s, s=0$ will achieve the optimum in (31). Overall, the consumer's post-trade bundle holding is $i+1-\max (1, \hat{p}(i+1, j))$ and the producer's post-trade bundle holding is $j+\max (1, \hat{p}(i+1, j))$.

Proof of Lemma 2. First, we rewrite the law of motion (3) in terms of "change" and "bundles" as follows:

$$
\begin{aligned}
& \pi_{k l+k^{\prime}}^{t+1}=\pi_{k l+k^{\prime}}^{t} \\
+ & \frac{1}{N} \sum_{i^{\prime}, i, j^{\prime}, j, i l+i^{\prime}>k l+k^{\prime}} \pi_{i l+i^{\prime}}^{t} \pi_{j l+j^{\prime}}^{t} \lambda^{t}\left(i l+i^{\prime}-k l-k^{\prime} ; i l+i^{\prime}, j l+j^{\prime}\right) \\
+ & \frac{1}{N} \sum_{i^{\prime}, i, j^{\prime}, j, j l+j^{\prime}<k l+k^{\prime}} \pi_{i l+i^{\prime}}^{t} \pi_{j l+j^{\prime}}^{t} \lambda^{t}\left(k l+k^{\prime}-j l-j^{\prime} ; i l+i^{\prime}, j l+j^{\prime}\right) \\
- & \frac{1}{N} \sum_{j^{\prime}, j} \pi_{k l+k^{\prime}}^{t} \pi_{j l+j^{\prime}}^{t} \sum_{p>0} \lambda^{t}\left(p ; v l+v^{\prime}, j l\right) \\
- & \frac{1}{N} \sum_{i^{\prime}, i} \pi_{i l+i^{\prime}}^{t} \pi_{k l+k^{\prime}}^{t} \sum_{p>0} \lambda^{t}\left(p ; i l, v l+v^{\prime}\right)
\end{aligned}
$$

where $\pi_{0}^{t}$ and $\pi_{B l}^{t}$ are given by two adding-up conditions (19). The conclusion is reached by differentiating this law of motion and evaluating the result at $\pi^{*}$. Because most of the terms in the law of motion are quadratic and $\pi_{i l+i^{\prime}}^{*}=0$ for $i^{\prime}>0$, after differentiating and evaluating them at $\pi^{*}$, many of the terms will disappear. Throughout this paper, the derivatives are evaluated at the steady state. We suppress such dependence to simplify the notations. One 
can show that for $\left(v^{\prime}, v\right) \neq\left(k^{\prime}, k\right)$ and $v^{\prime}, k^{\prime} \in \mathbb{L}$,

$$
\begin{aligned}
& \frac{\partial \pi_{k l+k^{\prime}}^{t+1}}{\partial \pi_{v l+v^{\prime}}^{t}} \\
= & \frac{1}{N} \sum_{j=0}^{B} \pi_{j l}^{*} \lambda^{*}\left(v l+v^{\prime}-k l-k^{\prime} ; v l+v^{\prime}, j l\right) \\
+ & \frac{1}{N} \sum_{i=0}^{B} \pi_{i l}^{*} \lambda^{*}\left(i l-k l-k^{\prime} ; i l, v l+v^{\prime}\right) \\
+ & \frac{1}{N} \sum_{j=0}^{B} \pi_{j l}^{*} \lambda^{*}\left(k l+k^{\prime}-j l ; v l+v^{\prime}, j l\right) \\
+ & \frac{1}{N} \sum_{i=0}^{B} \pi_{i l}^{*} \lambda^{*}\left(k l+k^{\prime}-v l-v^{\prime} ; i l, v l+v^{\prime}\right) \\
\geq & 0,
\end{aligned}
$$

and

$$
\begin{aligned}
& \frac{\partial \pi_{k l+k^{\prime}}^{t+1}}{\partial \pi_{k l+k^{\prime}}^{t}} \\
= & 1-\frac{1}{N} \sum_{i=0}^{B} \pi_{i l}^{*} \sum_{p>0, p \neq i l-k l-k^{\prime}} \lambda^{*}\left(p ; i l, k l+k^{\prime}\right) \\
& -\frac{1}{N} \sum_{j=0}^{B} \pi_{j l}^{*} \sum_{p>0, p \neq k l+k^{\prime}-j l} \lambda^{*}\left(p ; k l+k^{\prime}, j l\right) \geq 0 .
\end{aligned}
$$

Consider the case where $k^{\prime}>v^{\prime}$. Note that all the terms in (33) are associated with meetings where the total amount of change is $v^{\prime}$. By Lemma 1, nobody ends up with $k^{\prime}$ units of change after such meetings. Thus, all the terms are zero, and hence $\Psi_{\pi}$ in (22) has a block-triangular form.

To prove (ii) and (iii), we first establish several claims:

Claim 1 If $k^{\prime}<v^{\prime}$, then $\Psi_{\pi\left(v^{\prime}\right)}^{\pi\left(k^{\prime}\right)} \geq 0$. Moreover, the equality for the $(k, v)$ element holds if and only if for all $i \in \mathbb{B}$, meetings between il and $v l+v^{\prime}$ leave no one with $k l+k^{\prime}$ units of money with probability one. 
Claim 2 Each diagonal block is positive. Moreover, in the vth column of $\Psi_{\pi\left(v^{\prime}\right)}^{\pi\left(v^{\prime}\right)}$, the sum of elements is no greater than one, and it is equal to one if and only if for all $i \in \mathbb{B}$, meetings between il and $v l+v^{\prime}$ leave one agent with $v^{\prime}$ units of change with probability one.

Claim 3 The vth column of $\Psi_{\pi\left(v^{\prime}\right)}^{\pi\left(k^{\prime}\right)}$ for all $k^{\prime}=1, \cdots, v^{\prime}-1$ is equal to zero if and only if in the vth column of $\Psi_{\pi\left(v^{\prime}\right)}^{\pi\left(v^{\prime}\right)}$, the sum of elements is equal to one.

Claim 1 is exactly what equation (33) states. The first part of Claim 2 follows from the inequalities in (33) and (34). The second part is shown as follows:

$$
\begin{aligned}
& \text { [The sum of the } \left.v \text { th column of } \Psi_{\pi\left(v^{\prime}\right)}^{\pi\left(v^{\prime}\right)}\right] \\
& =\frac{\partial \pi_{v l+v^{\prime}}^{t+1}}{\partial \pi_{v l+v^{\prime}}^{t}}+\sum_{k=0, k \neq v}^{B-1} \frac{\partial \pi_{k l+v^{\prime}}^{t+1}}{\partial \pi_{v l+v^{\prime}}^{t}} \\
& =1-\frac{1}{N} \sum_{i=0}^{B} \pi_{i l}^{*} \sum_{p>0, p \neq i l-v l-v^{\prime}} \lambda^{*}\left(p ; i l, v l+v^{\prime}\right) \\
& +\frac{1}{N} \sum_{i=0}^{B} \pi_{i l}^{*} \sum_{k=0, k \neq v}^{B-1} \lambda^{*}\left(i l-k l-v^{\prime} ; i l, v l+v^{\prime}\right) \\
& +\frac{1}{N} \sum_{i=0}^{B} \pi_{i l}^{*} \sum_{k=0, k \neq v}^{B-1} \lambda^{*}\left(k l-v l ; i l, v l+v^{\prime}\right) \\
& -\frac{1}{N} \sum_{j=0}^{B} \pi_{j l}^{*} \sum_{p>0, p \neq v l+v^{\prime}-j l} \lambda^{*}\left(p ; v l+v^{\prime}, j l\right) \\
& +\frac{1}{N} \sum_{j=0}^{B} \pi_{j l}^{*} \sum_{k=0, k \neq v}^{B-1} \lambda^{*}\left(v l-k l ; v l+v^{\prime}, j l\right) \\
& +\frac{1}{N} \sum_{j=0}^{B} \pi_{j l}^{*} \sum_{k=0, k \neq v}^{B-1} \lambda^{*}\left(k l+v^{\prime}-j l ; v l+v^{\prime}, j l\right)
\end{aligned}
$$




$$
\begin{aligned}
=1 & -\frac{1}{N} \sum_{i=0}^{B} \pi_{i l}^{*} \sum_{p>0, p \neq i l-k l-v^{\prime} \text { or }(k-v) l \text { for } k \in \mathbb{B}} \lambda^{*}\left(p ; i l, v l+v^{\prime}\right) \\
& -\frac{1}{N} \sum_{j=0}^{B} \pi_{j l}^{*} \sum_{p>0, p \neq k l+v^{\prime}-j l \text { or }(v-k) l \text { for } k \in \mathbb{B}} \lambda^{*}\left(p ; v l+v^{\prime}, j l\right) .
\end{aligned}
$$

Note that $\sum_{p>0, p \neq i l-k l-v^{\prime} \text { or }(k-v) l \text { for } k \in \mathbb{B}} \lambda^{*}\left(p ; i l, v l+v^{\prime}\right)=0$ is equivalent to the fact that the meeting $\left(i l, v l+v^{\prime}\right)$ leaves no one with $v^{\prime}$ units of change with probability one. A similar statement holds for the second term in the above. Therefore we have Claim 2. Combining Claims 1 and 2 leads to Claim 3 .

(ii) In the following discussion, we denote the transpose of a vector by superscript $T$. For any $x \in \mathbb{N}$, we let $1_{x}=(1, \cdots, 1)^{T}$ and $0_{x}=(0, \cdots, 0)^{T}$, both of which are $x$-dimensional. Because $\Psi_{\pi}^{\pi}$ is an upper-triangular block matrix, the eigenvalues of $\Psi_{\pi}^{\pi}$ are those of $\Psi_{\pi\left(v^{\prime}\right)}^{\pi\left(v^{\prime}\right)}$, with $v^{\prime} \in \mathbb{L}$. Consider $\Psi_{\pi(1)}^{\pi(1)}$, the block corresponding to one-unit change. Claim 2 implies that in each column of $\Psi_{\pi(1)}^{\pi(1)}$, the sum of elements is equal to one: $1_{B}^{T} \Psi_{\pi(1)}^{\pi(1)}=1_{B}^{T}=1 \cdot 1_{B}^{T}$. Hence, $\Psi_{\pi(1)}^{\pi(1)}$ has a unit eigenvalue, and therefore, $\Psi_{\pi}^{\pi}$ has a unit eigenvalue.

(iii) Suppose by way of contradiction that $\Psi_{\pi}^{\pi}$ has an eigenvalue that is smaller than one, say $\tau \in(0,1)$, and that its associated eigenvector has only non-negative elements such that the law of motion could have exponential convergence to $\pi^{*}$ along this eigenvector. Denote that eigenvector by $\eta=$ $\left(\eta_{1}^{T}, \cdots, \eta_{l-1}^{T}\right)^{T} \geq 0$, where for each $v^{\prime} \in \mathbb{L}, \eta_{v^{\prime}}$ is a $B$-dimensional vector. We have $B(l-1)$ equations:

$$
0_{B(l-1)}=\left(\Psi_{\pi}^{\pi}-\tau I\right) \eta .
$$

Sum up the first $B$ equations, the second $B$ equations, etc.

$$
0_{l-1}=\left[\begin{array}{ccccc}
1_{B}^{T} \Psi_{\pi(1)}^{\pi(1)}-1_{B}^{T} \tau & 1_{B}^{T} \Psi_{\Pi(2)}^{\Pi(1)} & \cdots & & 1_{B}^{T} \Psi_{\pi(l-1)}^{\pi(1)} \\
0_{B}^{T} & 1_{B}^{T} \Psi_{\pi(2)}^{\pi(2)}-1_{B}^{T} \tau & \cdots & & \\
0_{B}^{T} & 0_{B}^{T} & \ddots & & \vdots \\
\vdots & \vdots & & \ddots & 1_{B}^{T} \Psi_{\pi(l-1)}^{\pi(l-2)} \\
0_{B}^{T} & 0_{B}^{T} & \cdots & & 1_{B}^{T} \Psi_{\pi(l-1)}^{\pi(l-1)}-1_{B}^{T} \tau
\end{array}\right]\left[\begin{array}{c}
\eta_{1} \\
\vdots \\
\vdots \\
\eta_{l-1}
\end{array}\right]
$$


By Claim 1, we have $1_{B}^{T} \Psi_{\Pi\left(v^{\prime}\right)}^{\Pi\left(k^{\prime}\right)} \geq 0$ for $k^{\prime}<v^{\prime}$ and thus the upper right blocks of the first matrix of the above equation are positive matrices. Moreover, the previous argument implies that $1_{B}^{T} \Psi_{\pi(1)}^{\pi(1)}-\tau 1_{B}^{T}=1_{B}^{T}-\tau 1_{B}^{T}=$ $(1-\tau) 1_{B}^{T}>0$. Therefore, we have $\eta_{1}=0$.

We use mathematical induction and assume that $\eta_{1}=\cdots=\eta_{v^{\prime}-1}=0$. In what follows, we want to show $\eta_{v^{\prime}}=0$. The following discussion is divided into two cases.

Case 1: Consider any $v \in \mathbb{B} \backslash B$ such that $\sum_{k=0}^{B-1} \frac{\partial \pi_{k l+v^{\prime}}^{t+1}}{\partial \pi_{v l+v^{\prime}}^{t}} \neq 1$. Then by Claim 3, there exists $k^{\prime}$ smaller than $v^{\prime}$ such that the $v$ th column of $\Psi_{\Pi\left(v^{\prime}\right)}^{\Pi\left(k^{\prime}\right)}$, which corresponds to the $\left(k^{\prime}, v^{\prime}\right)$ block in the first matrix of (35), has strictly positive elements. The sum of elements in this column $\sum_{k=0}^{B-1} \frac{\partial \pi_{k l+k^{\prime}}^{t+1}}{\partial \pi_{v l+v^{\prime}}^{t}}$ is strictly greater than 0 . If we look into the $k^{\prime}$ th row in the r.h.s. of (35), given that $\eta_{1}=\cdots=\eta_{v^{\prime}-1}=0$, the resulting vector is equal to $\sum_{r^{\prime}=v^{\prime}}^{l-1} 1_{B}^{T} \Psi_{\Pi\left(r^{\prime}\right)}^{\Pi\left(k^{\prime}\right)} \eta_{r^{\prime}}$. It satisfies the following condition:

$$
0=\sum_{r^{\prime}=v^{\prime}}^{l-1} 1_{B}^{T} \Psi_{\Pi\left(r^{\prime}\right)}^{\Pi\left(k^{\prime}\right)} \eta_{r^{\prime}} \geq 1_{B}^{T} \Psi_{\Pi\left(v^{\prime}\right)}^{\Pi\left(k^{\prime}\right)} \eta_{v^{\prime}} \geq \sum_{k=0}^{B-1} \frac{\partial \pi_{k l+k^{\prime}}^{t+1}}{\partial \pi_{v l+v^{\prime}}^{t}} \eta_{v^{\prime}}(v) \geq 0
$$

where $\eta_{v^{\prime}}(v)$ is the $v$ th element of vector $\eta_{v^{\prime}}$. The equality is by (35). The first inequality is because $1_{B}^{T} \Psi_{\Pi\left(r^{\prime}\right)}^{\Pi\left(k^{\prime}\right)} \eta_{r^{\prime}} \geq 0$ for all $r^{\prime} \geq v^{\prime}$. If we write out the matrix multiplication in $1_{B}^{T} \Psi_{\Pi\left(v^{\prime}\right)}^{\Pi\left(k^{\prime}\right)} \eta_{v^{\prime}}$, (33), (34) and $\eta_{v^{\prime}} \geq 0$ imply the second inequality. Since $\sum_{k=0}^{B-1} \frac{\partial \pi_{k l+k^{\prime}}^{t+1}}{\partial \pi_{v l+v^{\prime}}^{t}}$ is strictly greater than 0 , we must have $\eta_{v^{\prime}}(v)=0$.

Case 2: Consider any $v \in \mathbb{B} \backslash B$ such that $\sum_{k=0}^{B-1} \frac{\partial \pi_{k l+v^{\prime}}^{t+1}}{\partial \pi_{v l+v^{\prime}}^{t}}=1$. If we look into the $v^{\prime}$ th row in the r.h.s. of (35), the resulting vector is equal to $\left(1_{B}^{T} \Psi_{\Pi\left(v^{\prime}\right)}^{\Pi\left(v^{\prime}\right)}-\right.$ 
$\left.\tau 1_{B}^{T}\right) \eta_{v^{\prime}}+\sum_{r^{\prime}=v^{\prime}+1}^{l} 1_{B}^{T} \Psi_{\Pi\left(r^{\prime}\right)}^{\Pi\left(v^{\prime}\right)} \eta_{r^{\prime}}$. It satisfies

$$
\begin{aligned}
0 & =\left(1_{B}^{T} \Psi_{\Pi\left(v^{\prime}\right)}^{\Pi\left(v^{\prime}\right)}-\tau 1_{B}^{T}\right) \eta_{v^{\prime}}+\sum_{r^{\prime}=v^{\prime}+1}^{l-1} 1_{B}^{T} \Psi_{\Pi\left(r^{\prime}\right)}^{\Pi\left(v^{\prime}\right)} \eta_{r^{\prime}} \\
& \geq\left(1_{B}^{T} \Psi_{\Pi\left(v^{\prime}\right)}^{\Pi\left(v^{\prime}\right)}-\tau 1_{B}^{T}\right) \eta_{v^{\prime}} \\
& \geq\left(\sum_{k=0}^{B-1} \frac{\partial \pi_{k l+v^{\prime}}^{t+1}}{\partial \pi_{v l+v^{\prime}}^{t}}-\tau\right) \eta_{v^{\prime}}(v) \\
& \geq 0 .
\end{aligned}
$$

The equality is by (35). The first inequality is because $1_{B}^{T} \Psi_{\Pi\left(r^{\prime}\right)}^{\Pi\left(v^{\prime}\right)} \eta_{r^{\prime}} \geq 0$ for all $r^{\prime}>v^{\prime}$. If we write out the matrix multiplication in $\left(1_{B}^{T} \Psi_{\Pi\left(v^{\prime}\right)}^{\Pi\left(v^{\prime}\right)}-\right.$ $\left.\tau 1_{B}^{T}\right) \eta_{v^{\prime}}$, we have the sum over $\left(\sum_{k=0}^{B-1} \frac{\partial \pi_{k l+v^{\prime}}^{t+1}}{\partial \pi_{v l+v^{\prime}}^{t}}-\tau\right) \eta_{v^{\prime}}(v)$ for all $v \in \mathbb{B} \backslash B$. For those $v \in \mathbb{B} \backslash B$ such that $\sum_{k=0}^{B-1} \frac{\partial \pi_{k l+v^{\prime}}^{t+1}}{\partial \pi_{v l+v^{\prime}}^{t}} \neq 1$, we have $\eta_{v^{\prime}}(v)=0$ by Case 1 , and therefore $\left(\sum_{k=0}^{B-1} \frac{\partial \pi_{k l+v^{\prime}}^{t+1}}{\partial \pi_{v l+v^{\prime}}^{t}}-\tau\right) \eta_{v^{\prime}}(v)=0$ for such $v$. For those $v \in \mathbb{B} \backslash B$ such that $\sum_{k=0}^{B-1} \frac{\partial \pi_{k l+v^{\prime}}^{t+1}}{\partial \pi_{v l+v^{\prime}}^{t}}=1$, we have $\left(\sum_{k=0}^{B-1} \frac{\partial \pi_{k l+v^{\prime}}^{t+1}}{\partial \pi_{v l+v^{\prime}}^{t}}-\tau\right) \eta_{v^{\prime}}(v) \geq 0$. Combining these results for the two types of $v$ gives the second inequality. Since $\sum_{k=0}^{B-1} \frac{\partial \pi_{k l+v^{\prime}}^{t+1}}{\partial \pi_{v l+v^{\prime}}^{t}}-\tau$ is strictly greater than $0,(37)$ implies $\eta_{v^{\prime}}(v)=0$. Combining these two cases, we have $\eta_{v^{\prime}}=0$.

(iv) Because coefficient of $\lambda^{t}\left(p ; i l+i^{\prime}, j l+j^{\prime}\right)$ for $i^{\prime}+j^{\prime}>0$ in (3) is $\pm \frac{1}{N} \pi_{i l+i^{\prime}} \pi_{j l+j^{\prime}}$, it is equal to zero at the $l$-replica. Thus we have $\Psi_{\lambda}=0$.

Proof of Lemma 3. (i) Let $w_{x+p}-w_{x} \equiv \Delta w(x, p)$. The original Bellman equation can be written as

$$
\begin{gathered}
w_{i l+i^{\prime}}^{t}= \\
\sum_{j \in \mathbb{B} \backslash\{B\}, j^{\prime} \in \mathbb{L} \cup\{0\}} \frac{\pi_{j l+j^{\prime}}^{t} \sum_{p} \lambda^{t}\left(p ; i l+i^{\prime}, j l+j^{\prime}\right) u\left(\beta \Delta w^{t+1}\left(j l+j^{\prime}, p\right)\right)}{+\sum_{j \in \mathbb{B} \backslash\{B\}, j^{\prime} \in \mathbb{L} \cup\{0\}} \frac{\pi_{j l+j^{\prime}}^{t}}{N} \beta \sum_{p} \lambda^{t}\left(p ; i l+i^{\prime}, j l+j^{\prime}\right) w_{i l+i^{\prime}-p}^{t+1}+\frac{N-1+\pi_{B l}^{t} \beta w_{i l+i^{\prime}}^{t+1}}{N}}
\end{gathered}
$$


Recalling Lemma 1, we take the linear expansion of equation (38) at the steady state and then subtract $w_{i l}^{t}$ from $w_{i l+i^{\prime}}^{t}$ for $i^{\prime} \in \mathbb{L}$ to get the linear expansion of $\phi^{\Delta w}$ in (21):

$$
\begin{aligned}
& \sum_{\substack{j \in \mathbb{B} \backslash B \\
p \in \hat{p}(i, j) \\
v^{\prime} \in\left\{0, \cdots, i^{\prime}\right\}}} \frac{\pi_{j l}^{t}}{N} u^{\prime}\left(\beta \Delta w^{*}(j l, p l)\right) \beta \lambda^{*}\left(p l+v^{\prime} ; i l+i^{\prime}, j l\right) \Delta w^{t+1}\left(j l+p l, v^{\prime}\right) \\
& +\sum_{\substack{j \in \mathbb{B} \backslash B \\
p \in \hat{p}(i, j) \\
v^{\prime} \in\left\{0, \cdots, i^{\prime}\right\}}} \frac{\pi_{j l}^{*}}{N} \beta \lambda^{*}\left(p l+v^{\prime} ; i l+i^{\prime}, j l\right) \Delta w^{t+1}\left(j l-p l, i^{\prime}-v^{\prime}\right) \\
& +\frac{\pi_{B l}^{*}+N-1}{N} \beta\left(w_{i l+i^{\prime}}^{t+1}-w_{i l}^{t+1}\right) \\
& +\sum_{\substack{j l+j^{\prime} \notin\{0, B l\}\\
}} \kappa_{\left(i l+i^{\prime}, j l+j^{\prime}\right)}\left(\pi_{j l+j^{\prime}}^{t}-\pi_{j l+j^{\prime}}^{*}\right)
\end{aligned}
$$

where

$$
\begin{aligned}
\kappa_{\left(i l+i^{\prime}, j l+j^{\prime}\right)} \equiv & \frac{1}{N}\left\{\sum_{p} \lambda^{*}\left(p ; i l+i^{\prime}, j l+j^{\prime}\right)\left[u\left(\beta \Delta w^{*}\left(j l+j^{\prime}, p\right)\right)+\beta w_{i l+i^{\prime}-p}^{*}\right]\right. \\
& \left.-\sum_{p} \lambda^{*}\left(p ; i l, j l+j^{\prime}\right)\left[u\left(\beta \Delta w^{*}\left(j l+j^{\prime}, p\right)\right)+\beta w_{i l-p}^{*}\right]\right\} .
\end{aligned}
$$

By Lemma 1, $\kappa_{\left(i l+i^{\prime}, j l+j^{\prime}\right)}=0$ if $j^{\prime}=0$. Thus, the r.h.s. of (39) does not depend on $\pi_{F}^{t}$. Lemma 1 implies the same pure strategy in trading bundles across meetings $\left(i l+i^{\prime}, j l\right)$ for different change holding $i^{\prime}$. Thus, the r.h.s. depends on $w^{t+1}$ only throught incremental values $\Delta w^{t+1}$. Finally, note that because all trades in the support of randomization gives the same payoff, changing randomization has no impact on the current value. Therefore we have $\phi_{\lambda}=0$.

(ii) Looking into the coefficients with respect to $\pi_{N}^{t}$ in (39) gives $\phi_{\pi_{N}}^{\Delta w}$. Because of (7) and Lemma 1, two facts follow: (I) $\kappa_{\left(i l+i^{\prime}, j l+j^{\prime}\right)}=0$ for all $i^{\prime}, j^{\prime}$ such that $i^{\prime}+j^{\prime}<l$, and (II) as long as both $i^{\prime}+j^{\prime}$ and $i^{\prime \prime}+j^{\prime \prime}$ are greater than $l, \kappa_{\left(i l+i^{\prime}, j l+j^{\prime}\right)}=\kappa_{\left(i^{\prime \prime}+i l, j^{\prime \prime}+j l\right)}$. Therefore, it is valid to let $K_{i, j}$ be equal to $\kappa_{\left(i l+i^{\prime}, j l+j^{\prime}\right)}$ for $i^{\prime}+j^{\prime} \geq l$, and we have the statement. 
The strict positiveness of $K_{i, j}$ is due to the following. The first term of $K_{i, j}$ is the consumer's payoff in the meeting $\left(i l+i^{\prime}, j l+j^{\prime}\right)$, and the second term is that in the meeting $\left(i l, j l+j^{\prime}\right)$. Because $i^{\prime}+j^{\prime} \geq l, l-j^{\prime}$ units of money have the same value as a bundle to the producer. The consumer gets a higher payoff from giving that much change than she does in the meeting $\left(i l, j l+j^{\prime}\right)$.

(iii) Looking into the coefficients with respect to the incremental values of change gives $\phi_{\Delta w}^{\Delta w}$. Because the total amount of change in a meeting cannot increase after trade, we have

$$
\frac{d\left(w_{i l+i^{\prime}}^{t}-w_{i l}^{t}\right)}{d\left(w_{v l+v^{\prime}}^{t+1}-w_{v l}^{t+1}\right)}=0
$$

if $i^{\prime}<v^{\prime}$. Hence, all the blocks to the right of the diagonal are zero. For the diagonal blocks, we have

$$
\begin{aligned}
\left(\phi_{\Delta w_{i^{\prime}}}^{\Delta w_{i^{\prime}}}\right)_{i+1, v+1} & =\frac{d\left(w_{i l+i^{\prime}}^{t}-w_{i l}^{t}\right)}{d\left(w_{v l+i^{\prime}}^{t+1}-w_{v l}^{t+1}\right)} \\
& =\sum_{j \in \mathbb{B} \backslash B} \frac{\pi_{j l}^{*}}{N} u^{\prime}\left(\beta \Delta w^{*}(j l, v l-j l)\right) \beta \lambda^{*}\left(v l+i^{\prime}-j l ; i l+i^{\prime}, j l\right) \\
& +\sum_{j \in \mathbb{B} \backslash B} \frac{\pi_{j l}^{*}}{N} \beta \lambda^{*}\left(i l-v l ; i l+i^{\prime}, j l\right) \\
& +\frac{\pi_{B l}^{*}+N-1}{N} \beta \mathbf{1}\{i=v\} \\
& \geq 0,
\end{aligned}
$$

where the terms in the summation correspond to inflows into $v l+i^{\prime}$ generated by meetings $\left(i l+i^{\prime}, j l\right)$ for $j \in \mathbb{B} \backslash B$.

Proof of Lemma 4. By Lemmas 2(i)(iv) and 3(i), the stacked system (26)-(27) when linearized does not depend on $\lambda^{t}$, and its Jacobian has the following form:

$$
\left[\begin{array}{cccc}
\Psi_{\pi_{N}}^{\pi_{N}} & O & O & O \\
-\left[\phi_{\Delta w}^{\Delta w}\right]^{-1} \phi_{\pi_{N}}^{\Delta w} & {\left[\phi_{\Delta w}^{\Delta w}\right]^{-1}} & O & O \\
\Psi_{\pi_{N}}^{\pi_{F}} & O & \Psi_{\pi_{F}}^{\pi_{F}} & O \\
* & * & * & *
\end{array}\right]
$$

where the $*$ s are blocks irrelevant to our analysis. This implies that $\left(\pi_{N}^{t}, \Delta w^{t}\right)$ does not depend on other variables locally and their linear system is repre- 
sented by (28). Finally, since the upper-right block of $A$ is a zero matrix, the eigenvalues of $A$ are those of $\Psi_{\pi_{N}}^{\pi_{N}}$ and $\left[\phi_{\Delta w}^{\Delta w}\right]^{-1}$. By Lemma 2(ii), $\Psi_{\pi_{N}}^{\pi_{N}}$ has a unit eigenvalue.

Proof of Lemma 5. (i) Suppose $\eta=\left(z, \eta_{2}\right)$ is the eigenvector of $A$ associated with a unit eigenvalue of $\Psi_{\pi_{N}}^{\pi_{N}}$ :

$$
\left[\begin{array}{cc}
\Psi_{\pi_{N}}^{\pi_{N}} & 0 \\
-\left[\phi_{\Delta w}^{\Delta w}\right]^{-1} \phi_{\pi_{N}}^{\Delta w} & {\left[\phi_{\Delta w}^{\Delta w}\right]^{-1}}
\end{array}\right]\left[\begin{array}{c}
z \\
\eta_{2}
\end{array}\right]=\left[\begin{array}{c}
z \\
\eta_{2}
\end{array}\right]
$$

Hence, we have $-\left[\phi_{\Delta w}^{\Delta w}\right]^{-1} \phi_{\pi_{N}}^{\Delta w} z+\left[\phi_{\Delta w}^{\Delta w}\right]^{-1} \eta_{2}=\eta_{2}$. Note that the inverse of the lower-triangular matrix is a lower-triangular matrix. Therefore, we have

$\eta_{2}=\left(I-\phi_{\Delta w}^{\Delta w}\right)^{-1} \phi_{\pi_{N}}^{\Delta w} z=\left[\begin{array}{c}0 \\ \vdots \\ 0 \\ \left(I-\phi_{\Delta w_{l-1}}^{\Delta w_{l-1}}\right)^{-1} K z_{1}\end{array}\right]+\cdots+\left[\begin{array}{c}\left(I-\phi_{\Delta w_{1}}^{\Delta w_{1}}\right)^{-1} K z_{l-1} \\ * \\ \vdots \\ *\end{array}\right]$.

(ii) Assume by way of contradiction that $\left(\varphi_{\Delta w_{i^{\prime}}}\right)_{i}=0$ for some $i$. By the definition of $\varphi$, We have

$$
\left(I-\phi_{\Delta w_{i^{\prime}}}^{\Delta w_{i^{\prime}}}\right) \varphi_{\Delta w_{i^{\prime}}}=K z_{l-i^{\prime}}
$$

where the r.h.s. is strictly positive because of $z_{l-i^{\prime}} \geq 0$ and Lemma 3 (ii). The $i$-th element of the l.h.s. of (42) is $\left.-\sum_{v \neq i}\left(\phi_{\Delta w_{i^{\prime}}}^{\Delta w_{i, v}}\right)_{i \Delta w_{i^{\prime}}}\right)_{v}$ which cannot be strictly positive because $\left(\phi_{\Delta w_{i^{\prime}}}^{\Delta w_{i^{\prime}}}\right)_{i, v}$ is non-negative for $v \neq i$ (Lemma 3 (iii)).

(iii) Suppose by contradiction $\varphi_{\Delta w_{i^{\prime}}} \geq 0$. (ii) implies $\varphi_{\Delta w_{i^{\prime}}}>0$. By letting $i=0$ and $\lambda^{*}\left(i^{\prime} ; i^{\prime}, 0\right)=1$ in (40), it can be shown that the first element of $\left(I-\phi_{\Delta w_{i^{\prime}}}^{\Delta w_{i^{\prime}}}\right) \varphi_{\Delta w_{i^{\prime}}}$ is

$$
\left[1-\beta\left(\frac{\pi_{B l}^{*}+N-1+\pi_{0}^{*} u^{\prime}(0)}{N}\right)\right]\left(\varphi_{\Delta w_{i^{\prime}}}\right)_{1}-\sum_{v=1}^{B-1}\left(\phi_{\Delta w_{i^{\prime}}}^{\Delta w_{i^{\prime}}}\right)_{i+1, v+1}\left(\varphi_{\Delta w_{i^{\prime}}}\right)_{v+1}<0
$$

since $u^{\prime}(0)$ is assumed to be any large number. $\left(u^{\prime}(0)>[N / \beta-N+(1-\right.$ $\left.\left.\pi_{B l}^{*}\right)\right] / \pi_{0}^{*}$ is sufficient here. This condition resembles (13) in the example section.) However, the strict inequality (43) contradicts the fact that the r.h.s. of (42) is strictly positive. 


\section{References}

[1] Camera, G., Corbae, D. (1999): "Money and price dispersion," International Economic Review, Vol. 40, No. 4, 985-1008

[2] Deviatov, A. (2006) : "Money creation in a random matching model," Topics in Macroeconomics, 6, No. 3, article 5

[3] Green, E. J., Zhou, R. (2002): "Dynamic monetary equilibrium in a random matching economy," Econometrica, Vol. 70, No. 3, 929-969

[4] Huang, P., Igarashi, Y. (2012): "Trejos-Wright with a 2-unit bound: existence and stability of monetary steady states," unpublished manuscript

[5] Lomeli, H., Temzelides, T. (2002): "Discrete time dynamics in a random matching monetary model," Economic Theory, 20, 259-269

[6] Luenberger, D. G. (1979): Introduction to Dynamic Systems: Theory, Models, and Applications.

[7] Lucas, R.,Prescott, E., Stokey, N. (1989): Recursive Methods in Economic Dynamics.

[8] Molico, M. (2006): "The distribution of money and prices in search equilibrium," International Economic Review, Vol. 47, No. 3, 701-722

[9] Shi, S. (1995): "Money and prices: a model of search and bargaining," Journal of Economic Theory, 67, 467-496

[10] Strogatz, S. H. (1994): Nonlinear Dynamics and Chaos with Application to Physics, Biology, Chemistry, and Engineering.

[11] Trejos, A., Wright, R. (1995): "Search, bargaining, money and prices," Journal of Political Economy, Vol. 103, No. 1, 118-141

[12] Wallace, N. (2014): "Optimal money creation in "pure currency" economies: a conjecture," The Quarterly Journal of Economics, 129, 259-274

[13] Wallace, N., Zhu, T. (2004): "A commodity-money refinement in matching models," Journal of Economic Theory, 117, 246-258 
[14] Zhu, T. (2003): "Existence of a monetary steady state in a matching model: indivisible money," Journal of Economic Theory, 112, 307-324 